\title{
MY09/MY11 PCR Assay
}

National Cancer Institute

\section{Source}

National Cancer Institute. MY09/MY11 PCR Assay. NCI Thesaurus. Code C120296.

A PCR technique that uses the MY09/MY11 degenerate primer set for HPV detection. It amplifies a 450 bp region from the L1 region of the HPV genome. 